\title{
An Agent Based-Model and Equilibrium Analysis of Academic P\&T Decisions: The Effects of Inbreeding
}

\author{
Sanjoy Das
}

\begin{abstract}
In academic institutions, merit based promotion \& tenure decisions have always been beset with controversy. This paper suggests an agent based model of the decision making process using spectral graph theory, where the voting agents are the vertices of the graph, and edge weights are determined based on the extent of collaborative research between the agents, as well as their estimated levels of social interactions. The model assumes that agents with lower research productivities tend to interact more often with one another. Using the graph theoretic spectrum, the paper applies a multidimensional representation that maps the voting agents into points on a low-dimensional grid, where agents that are likely to influence each other more are closely spaced. A multi-agent system model is proposed, where votes are determined based on very small randomly assigned initial values, and the mutual interaction during the decision making process. The model incorporates limited collusive voting within academically inbred agents. The proposed model is able to accurately reproduce a known promotion decision making from a department of a research oriented university which involved a sizable number of voting agents with low research output.
\end{abstract}

Index Terms-Con vex function, graph laplacian, promotion and tenure, multi-agent systems, simulations.

\section{INTRODUCTION}

Academic promotion and tenure $(\mathrm{P} \& \mathrm{~T})$ criteria can be broadly divided into two categories, merit based and seniority based [1]. American universities have invariably adopted merit based P\&T criteria. A well devised merit based P\&T policy can have a very positive impact on the department, leading to higher academic productivity [2]. To the best of author's knowledge, there is only one such study that models the P\&T decision making process [3]. This investigation, which is based on game theory, is largely confined to only two-member committees.

A novel multi-agent system (MAS) model a departmental P\&T committee using spectral graph theory [4] is proposed in this research. The P\&T committee and the candidate (referred to as agents for the remainder of this paper) are represented as the vertices of a weighted, undirected graph. There are edges connecting every pair of vertices; their weights reflecting the levels of academic as well as social interaction between the corresponding agents. The approach assumes that the degree to which agents influence each other is determined by their interactions, both research collaborations as well as miscellaneous academic and social bonds.

Manuscript received January 15, 2018; revised May 10, 2018

S. Das is with the ECE Department, Kansas State University, Manhattan, KS 66506, USA (e-mail: sdas@ksu.edu).
Academic inbreeding, i.e. the presence of faculty with degrees from the same department, is known to have a deleterious effect on the department [5]-[8]. Academic inbreeding in also considered in this research. Using spectral embedding, the graph's vertices are mapped into points in a two-dimensional plane where distances between vertices depends inversely on the degrees of influence that the as sociated agents have upon one another.

Next, a quadratic objective function is proposed that uses the graphical representation of the model [9], [10]. The agents, voting decisions are treated as a real valued vector. This objective function takes into account two factors: that the voting decision of each agent are mutually influenced by each other during the actual voting process, and that each agent assumes a small initial position where less productive agents are more randomized than the more productive ones, in terms of research output.

The results described here are based on the voting outcome of an actual department, which is kept confidential. From the actual experience of the candidate, the votes cast by the agents in that $P \& T$ committee have been estimated to a reasonably high degree of confidence. Official correspondence from competent authorities outside the department establish that the candidate merited a positive outcome, based on his/her research accomplishments.

In the model described here, a high degree of emphasis is based on the candidates' research publications [11]; consequently, productivity levels of not only the candidate agent, but also those of the P\&T committee is based on the numbers of their scholarly publications. The funding levels of individual agents is also taken into account. The edge weights are determined in a similar manner, based on account research paper co-authorship and joint funding. The productivy metric is consistent with faculty seniority, in that particular department, with the so called 'academic deadwood' being the least productive which is also the usual case [12], [13].

It has been shown that reasonable values of the parameters can recreate the outcome of the P\&T decision with remarkable precision, thereby validating the model. The robustness of the parameters are also established here. Consistent with recent findings that inbred faculty tend to favor each other [14], small increments in the weights linking inbred agents allowed the model to reproduce the outcome to full accuracy. As one study reports the role of department leadership in productivity, although not specifically outcomes of such decision making [5], [16], the role of that department's head in ensuring a fairer voting outcome has been briefly considered. 


\section{APPROACH}

\section{A. Graph Construction}

Academic scholarship can be determined based on a variety of factors, with research publications being the most common criterion. Other criteria include the total extramural support, the number of graduate students supervised, journal editorship, etc. Let $\operatorname{CRIT}_{k}(i)$ denote the output level (e.g. publication count) of agent $i$ for criterion $k$. The weighted productivity level of agent $i$ is given by the expression below.

$$
p_{i}=\sum_{k} \beta_{k} \frac{\operatorname{CRIT}_{k}(i)}{\sum_{j} \operatorname{CRIT}_{k}(j)} .
$$

In the above expression, each $\beta_{k}$ is a parameter that determines the weights placed on the criterion $k$ in evaluating the overall productivity.

The summation in the denominator is carried out over all agents in the department, and is used to normalize the measure, so that each term in the summation in (1) never exceeds unity, as well as to ensure that the relative weight of criterion $k$ is entirely determined by the value assigned to the corresponding numerical value of $\beta_{k}$. The productivity vector $\mathbf{p}$ is defined as the $N \times 1$ vector whose entries are $p_{i}$ and $N$ is the total number of agents.

With $\operatorname{CRIT}_{k}(i, j)$ quantifying the level of collaboration between agents $i$ and $j$ in terms of criterion $k$, the overall level of research and other academic collaboration, $R_{i j}$, is obtained using the following expression.

$$
R_{i j}= \begin{cases}\sum_{k} \alpha_{k} \frac{\operatorname{CRIT}_{k}(i, j)}{\sum_{j} \operatorname{CRIT} T_{k}(j)}, & i \neq j \\ 0, & i=j .\end{cases}
$$

For simplicity, the MAS model assumes that is the amount of time that each agent $i$ devotes to scholarly work is directly proportional to the productivity, $p_{i}$. The remaining time can be spent in meetings as well as non-academic collaborations. Assuming that the maximum time spent by an individual within the department is $P_{\max }$, the quantity $S_{i j}$ below determines the level of social interactions between agents $i$ and $j$.

$$
S_{i j}= \begin{cases}P_{\max }-\eta \sqrt{p_{i} p_{j}}, & i \neq j \\ 0, & i=j .\end{cases}
$$

The quantity $\eta<1$ is a model parameter called the socialization constant. The other parameter, $P_{\max }$ must be high enough relative to $\eta$, so that for every pair of agents $i$ and $j, W_{i j}>0$. Negative values for the weights $W_{i j}$ are not usually allowed in graphs, and $W_{i j}=0$ would result in a graph that is not fully connected. The $N \times N$ matrices $\mathbf{R}=\left[R_{i j}\right]$ and $\mathbf{S}=\left[S_{i j}\right]$ are obtained using the expressions in (2) and (3) above.

Let $\mathcal{J}$ be the set of academically inbred agents. The vector $\mathbf{u}$ is defined as,

$$
u_{i}= \begin{cases}0, & i \in \mathcal{J} \\ 1 & i \notin \mathcal{J}\end{cases}
$$

The weight matrix $\mathbf{W}=\left[W_{i j}\right]$ of the underlying graph is given by the expression below,

$$
\mathbf{W}=\mathbf{R}+\mathbf{S}+\gamma \mathbf{u} \mathbf{u}^{\mathrm{T}} .
$$

The quantity $\gamma$ is another model parameter called the inbreeding constant. To neglect any extra amount of influence that agents in $\mathcal{J}$ may exert upon one another, the value of $\gamma$ may be optionally set to zero, although a small positive value is suggested. The Laplacian $\mathbf{L}$ of the graph is obtained as follows [4].

$$
L_{i j}=\left\{\begin{aligned}
-W_{i j}, & i \neq j \\
\sum_{k} W_{i k}, & i=j .
\end{aligned}\right.
$$

With $N$ being the total number of agents, $\mathbf{W}$ and $\mathbf{L}$ are $N \times N$ symmetric matrices. Moreover, the Laplacian matrix $\mathbf{L}$ is positive semi-definite, i.e. $\mathbf{L} \geqslant \mathbf{0}$. When $P_{\max }$ is sufficiently high, the under graph is fully connected. In such a case it can be shown that $\mathbf{L}$ spans an $N-1$ subspace, so that $\operatorname{rank}(\mathbf{L})=N-1$ [4].

\section{B. Multi-Agent System}

In a typical P\&T decision process, some of the agents in the committee enter the process having determined a priori their voting decision. Their participation is restricted to influence the votes of the undecided agents [3]. Accordingly, the MAS model distinguishes between three sets of agents, $\mathcal{V}^{-}$and $\mathcal{V}^{+}$are the sets of agents with prior decisions to vote against, and in favor of the candidate. The set of undecided agents, $\mathcal{V}_{\mathrm{U}}$ comprises of all remaining agents. Note that the candidate agent $c$ is not included in any of these sets, $\mathcal{V}^{-}$, $\mathcal{V}^{+}$or $\mathcal{V}_{\mathrm{U}}$.

The votes cast by each undecided agent is based on a decision variable $x_{i}$. When $x_{i}$ is positive, the agent $i$ votes in favor of the candidate, and when it is negative, the agent votes against the latter. For agents in $\mathcal{V}^{-}$and $\mathcal{V}^{+}$the decision variable is only used to influence the undecided agents. Prior to the decision process, each agent's decision variable in $\mathcal{V}_{\mathrm{U}}$ is assigned a small random value $x_{i}^{0}$. For the agents in $\mathcal{V}^{-}$and $\mathcal{V}^{+}$, the values of $x_{i}^{0}$ are set to -1 and +1 . For the undecided agents, the initial value is obtained in the following manner.

$$
x_{i}^{0}=\alpha\left(\frac{p_{i}}{P_{\max }} m_{c}+\left(1-\frac{p_{i}}{P_{\max }}\right) r\right), \quad i \in \mathcal{V}_{\mathrm{U}} .
$$

In the above expression, the quantity $r$ is a uniformly distributed random variable ly ing in the range -1 to +1 (i.e. $r \sim U[-1,+1])$ and $\alpha$ is a small positive constant that determines the maximum randomness. The quantity $m_{c} \in[-1,+1]$ quantifies the overall merit of the candidate.

The factor appearing within parenthes is to the right of the above equation contains two terms. The first term is directly proportional to $p_{i}$ so an undecided agent with a higher productivity places greater emphasis on the candidate's own merit worthiness. The second term allows the votes cast by agents with lower productivities acquire more random initial values. It should be noted that the only role of the noise parameter $\alpha$ is to determine the range of initial voting assignments $x_{i}^{0}$ of the undecided agents in $\mathcal{V}_{\mathrm{U}}$. 
For a deserving candidate the quantity $m_{c}$ can be set to a value of at most +1 ; conversely for an undeserving candidate $m_{c}$ can be assigned a value of no less than -1 . Unless the merit of the candidate can be determined with a very high degree of confidence through extraneous means, a suggested way to assign a value to $m_{c}$ is according to either of the following two expressions provided below.

$$
m_{c}=\frac{1}{P_{\max }}\left(p_{c}-\frac{1}{2} P_{\max }\right)
$$

or,

$$
m_{c}=\frac{1}{P_{\max }}\left(p_{c}-\frac{1}{2 N} \sum_{i} p_{i}\right)
$$

The final value of the decision variables is determined in accordance with the expression shown below.

$$
\mathbf{x}_{\infty}=((1+\varepsilon) \mathbf{I}+\mu \mathbf{L})^{-1} \mathbf{x}_{0} .
$$

The quantities $\mu$ and $\varepsilon$ above are the influence and regularization constants. The influence constant sets the degree to which agents' decisions are decided through mutual interactions, relative to their random initial assignments. It can be shown that the above expression for $\mathbf{x}$ minimizes the following cost function.

$$
\varphi(\mathbf{x})=\frac{1}{2}\left(\left\|\mathbf{x}-\mathbf{x}_{0}\right\|^{2}+\varepsilon(\mathbf{x}-\mathbf{1})^{\mathrm{T}}(\mathbf{x}+\mathbf{1})+\mu \mathbf{x}^{\mathrm{T}} \mathbf{L x}\right) .
$$

The first term within parenthesis appearing to the right of the above expression for the cost function acquires a minimu m value of when none of the agents in the committee deviate from their initially assumed values, i.e. when $\mathbf{x}=\mathbf{x}_{0}$. This it minimizes the deviations of the agents' decisions from their initial values. The second term is zero when each $x_{i}$ is either +1 or -1 . It is optional and can be included if the voting outcomes be close to \pm 1 . Simulations indicate that the relative outcome is not affected even with the constant $\varepsilon$ being unity, although a sufficiently small value is recommended.

The third term in the expression for $\varphi(\mathbf{x})$ involves the Laplacian L. It can be shown that it simplifies as follows [4, $15]$.

$$
\mathbf{x}^{\mathrm{T}} \mathbf{L} \mathbf{x}=\frac{1}{2} \sum_{i} \sum_{j \neq i} W_{i j}\left(x_{i}-x_{j}\right)^{2}
$$

The above expression shows that $\mathbf{x}^{\mathrm{T}} \mathbf{L x}$ is the sum of the squared difference between the decisions of every pair of agents $i$ and $j$, weighted by $W_{i j}$ which depicts the amount of influence they have on each other. Thus, each term tries to keep the voting outcomes of agents that exert more influence on each other, to remain close. The quantity $\mu$ is termed the mutual influence constant.

The equilibrium value $\mathbf{x}_{\infty}$ can be obtained in the following manner. When $\|\cdot\|$ is the Euclidean norm, the first term to the right of (12) is equal to $\left(\mathbf{x}-\mathbf{x}_{0}\right)^{\mathrm{T}}\left(\mathbf{x}-\mathbf{x}_{0}\right)$, the derivative of $\varphi(\mathbf{x})$ in (12) is given by,

$$
\nabla \varphi(\mathbf{x})=\mathbf{x}-\mathbf{x}_{0}+\varepsilon(\mathbf{x}-\mathbf{1})+\mu \mathbf{L x} .
$$

The equilibriu $\mathrm{m}$ value can be obtained by letting $\nabla \varphi(\mathbf{x})=$ $\mathbf{0}$ when $\mathbf{x}=\mathbf{x}_{\infty}$. The Hessian of $\varphi(\mathbf{x})$ is,

$$
\nabla^{2} \varphi(\mathbf{x})=(1+\varepsilon) \mathbf{I}+\mu \mathbf{L} .
$$

Here, $\mathbf{I}$ is the identity matrix. It can be seen that $\mathbf{x}^{\mathrm{T}} \mathbf{L x} \geq 0$ since the right of the equality in (12) is quadratic. Whence, $\nabla^{2} \varphi(\mathbf{x}) \geqslant \mathbf{0}$ so that,

$$
\mathbf{x}_{\infty}=\underset{\mathbf{x}}{\operatorname{argmin}} \varphi(\mathbf{x}) .
$$

The results obtained from this equilibrium analysis is presented in the next section.

\section{RESULTS AND DISCUSSION}

\section{A. Scenario}

As the scenario considered here pertained to the promotion decision of a candidate, multiple years were involved. Hence, the voting decisions of the committee members could be estimated to a very high degree of confidence. There were 11 agents, labeled $1-11$, including the candidate $(c=1)$. The department head $(h=4)$ was absent from the P\&T committee, which consisted of the remaining 9 agents, so that $\mathcal{V}=\{2,3,5,6,7,8,9,10,11\}$. The agents in the $\mathrm{P} \& \mathrm{~T}$ committee who made their decisions prior to the committee consisted of two supporting agents, who have a strong history of collaborative research with the candidate $(7,8)$, and two malevolent agents $(6,11)$ who cast negative votes. The set of academically inbred agents who had received at least one degree from the same department was $\mathcal{J}=\{2,3,4,11\}$.

The justification that was provided by agents 6 and 11 for their negative votes is considered in this research as not suitable grounds for that department's P\&T decisions because of three reasons: $(i)$ the posited rationale they provided were not listed in the department's P\&T guidelines, (ii) the candidate's research was subsequently found to meet or exceed the promotion criteria by more competent, higher level authority outside the department, and, significantly, (iii) the previous year, another candidate, agent 10 , had been promoted without meeting the same requirements, and with both 6 and 11 voting in favor. For these reasons, the candidate has been considered as meriting a positive outcome wherever needed in the results described later. Additionally, it should be noted that the productivities, $p_{6}=0.789$ and $p_{11}=0.727$ of agents 6 and 11 (see next section) were found to be relatively low in comparison to others, and based on their productivities as well as retirement status, agents 6 and 11 may be considered academic deadwood [12]. Conversely, that of agents 7 and 8 were among the most productive in the department $\left(p_{7}=\right.$ 4.087 and $p_{8}=2.769$ ).

Furthermore, agent 10 who had been successfully promoted the previous year, also voted against the candidate, agent 1's P\&T decision, despite being clearly aware that the rationale being set forth by agents 6 and 11, during the P\&T meeting were not appropriate reasons. This agent (10) is classified as a strategic agent - the equivalent of a zero-sum player within a game theoretic context. Agent 10 also subsequently received academic recognition for which the 
candidate, agent 1 , would have also qualified. It is recommended that without strong underlying reasons, such an agent should be included within the set of undecided agents.

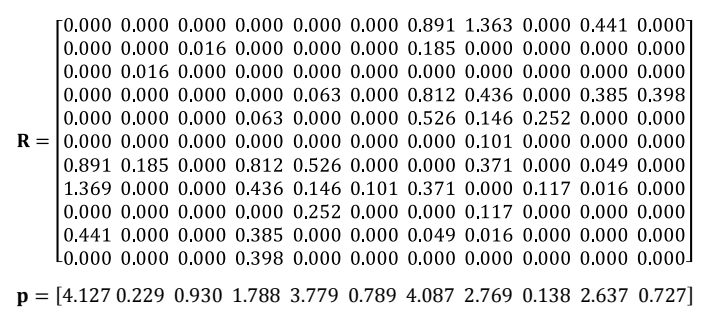

Fig. 1. The values of the entries of vector $\mathbf{p}$ and matrix $\mathbf{R}$.

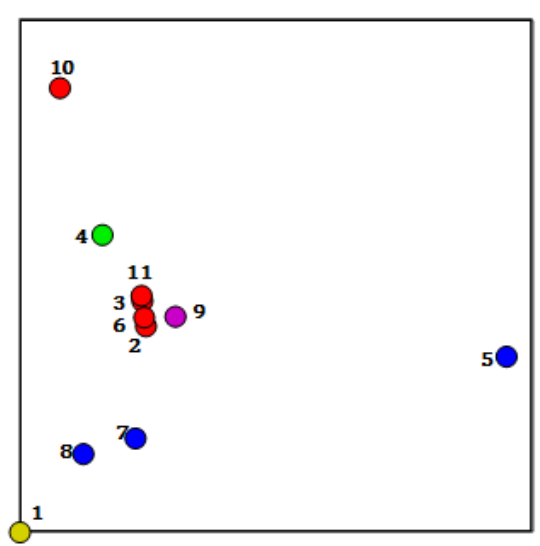

Fig. 2. Two-dimensional embedding with the following values: $\eta=0.25$, $P_{\max }=7$, and $\gamma=0.25[8]$.

Although to the best of the author's knowledge, the voting decisions of all agents as well as their underlying justifications, as described earlier, are authentic, the simulations below are generalized enough to be able to recreate any other similar $\mathrm{P} \& \mathrm{~T}$ decision process with a high degree of fidelity. The parameters used in the MAS model were few, and they were assigned reasonable numerical values (sometimes zero). The model was found to be very robust to changes in these values.
The final voting sets obtained is this manner were the set of malevolent and strategic agents, $\mathcal{V}^{-}=\{6,10,11\}$, the set of supportive agents, $\mathcal{V}^{+}=\{7,8\}$, and the set of undecided agents $\mathcal{V}_{\mathrm{U}}=\{2,3,5,9\}$, which includes the graduate program coordinator $(g=9)$. The simulations detailed below focus on how these four prior undecided agents arrived at their eventual decisions.

\section{B. Graph Weights}

If $J R(i), P B(i)$, and $F D(i)$ represent the total number of journal articles published, total number of publications, and the total grant level by agent $i$ during that period, its productivity level was computed as follows.

$$
p_{i}=\frac{2 J R(i)}{3 \sum_{k} J R(k)}+\frac{P B(i)}{3 \sum_{k} P B(k)}+\frac{F D(i)}{\sum_{k} F D(k)} .
$$

To account for administrative responsibilities of the department chair $(h=4)$ and the graduate coordinator $(g=9)$, their productivities were incremented, so that the adjusted productivities were,

$$
p_{i}= \begin{cases}p_{i}+2, & i=h \\ p_{i}+1, & i=g \\ p_{i}, & \text { otherwise }\end{cases}
$$

These adjusted values were used in all simulations described earlier in [16] as well as in this research.

With $J R(i, j)$ and $P B(i, j)$ denoting the total number of published journal articles and all articles co-authored by any pair of agents $i$ and $j$, and $F D(i, j)$ being their joint funding, the academic and research collaboration between the $m$ was quantified in the following manner.

$$
R_{i j}=\frac{2 J R(i, j)}{3 \sum_{k} J R(k)}+\frac{P B(i, j)}{3 \sum_{k} P B(k)}+\frac{F D(i, j)}{\sum_{k} F D(k)} .
$$

Figure 1. shows the values of the matrix $\mathbf{R}=\left[R_{i j}\right]$ and vector $\mathbf{p}=\left[p_{i}\right]$ that were used in this analysis. These are identical to those used in the earlier study [8].
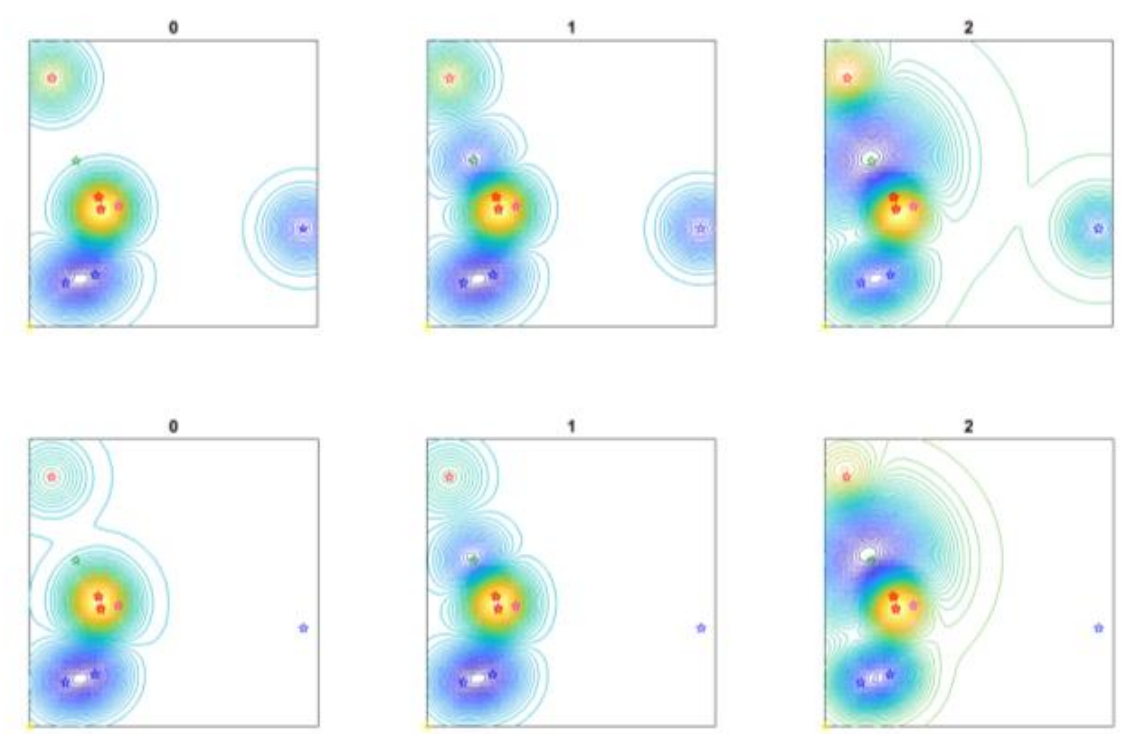

Fig. 3. Embedding showing how an effective department chair may influence the outcome of the decision process with the following values of the constants: $\eta=0.25, P_{\max }=7, \gamma=0.25$. The quantity $\sigma=4 \times 10^{-4}$ for all agents except the department chair (4) which was $\sigma=4 \times 10^{-4}($ left and middle columns) and $\sigma=16 \times 10^{-4}$ (right column). 

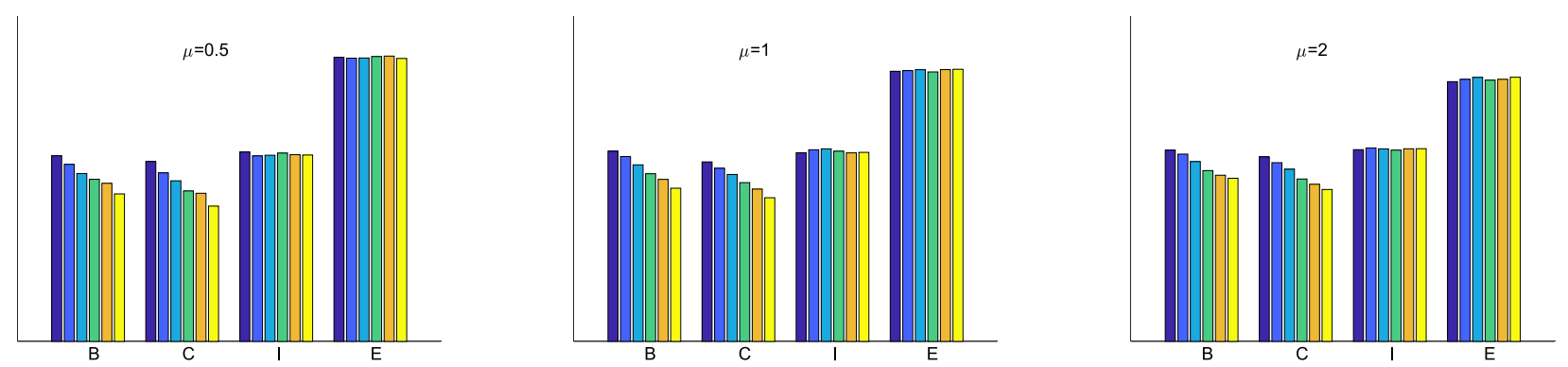

Fig. 4. Hist ograms showing the average votes of the undecided candidates, sorted in order of productivity with the following value s: $\mu=0.5$ (left), $\mu=1.0$ (middle) and $\mu=2.0$. (right). The inbreeding parameter $\gamma$ were at values $\gamma=0,0.2,0.4,0.6,0.8,1.0$.

A two-dimensional embedding is a plot of $\lambda_{2}^{-1} \mathbf{v}_{2}$ (x-axis) vs. $\lambda_{3}^{-1} \mathbf{v}_{3}$ (y-axis). The coordinates of agent $i$ in this plot are the $i^{\text {th }}$ entries of the vectors $\lambda_{2}^{-1} \mathbf{v}_{2}$ and $\lambda_{3}^{-1} \mathbf{v}_{3}$.

Fig. 2 is the two-dimensional embedding of the graph, with $\eta=0.25, P_{\max }=7$, and $\gamma=0.25$ that were used in all remaining simulations. The value $P_{\max }=7$ can be interpreted as the productivity of a hypothetical agent who works seven days a week.

All points representing the agents are color coded for better visualization. The candidate (agent 1) is shown in yellow (bottom left). The agents in $\mathcal{V}^{+}$as well as agent 9 (included in $\mathcal{V}_{\mathrm{U}}$ ) who voted favorably appear in blue, whereas the agents that voted against are in red.

The department chair who did not participate is shown in green. Agent 9, who voted in favor, and against the candidate in two separate instances, is shown in magenta.

The proximity of the points representing the undecided agents 2 and 3 to two malevolent agents 6 and 11 clearly shows how agents 2 and 3 voted negatively. This voting strategy has been interpreted to be, at least in part, a result of collusion [16]. Agent 9 is at a higher distance, and consequently did not vote negatively always. Agent 5 who is placed at a significantly larger distance, was clearly not influenced by the malevolent agents, explaining its eventual decision to cast a vote in favor.

Although the chair did not participate in the $\mathrm{P} \& \mathrm{~T}$ process, its potential role in influencing the other agents can be investigated by enhancing the two-dimensional embedding by quantifying the amount of influence any agent $i$ with coordinates $\mathbf{z}_{i}$ exerts at any other point $\mathbf{z}$ in the plot. For example, if $x_{i}$ is the voting decision of each agent $i$, the net influence $x(\mathbf{z})$ at $\mathbf{z}$ of all agents can be formu lated using the following expression.

$$
x(\mathbf{z})=\sum_{i} x_{i} e^{\frac{1}{\sigma}\left\|\mathbf{z}-\mathbf{z}_{i}\right\|^{2}} .
$$

In the above expression, $\left\|\mathbf{z}-\mathbf{z}_{i}\right\|$ is the Euclidean distance between the two dimensional points $\mathbf{z}$ and $\mathbf{z}_{i}$, and the quantity $\sigma$ is a small parameter that can be adjusted for best visualization.

Fig. 3 shows contour plots where the agents in $\mathcal{V}^{+}$are assigned voting decisions $x_{i}=+1$ while those in $\mathcal{V}^{-}$, $x_{i}=-1$. For the undecided agents in $\mathcal{V}_{\mathrm{U}}, x_{i}=0$. The contours are based on Eq. (19) with $\sigma=4 \times 10^{-4}$.

The decision $x_{4}$ of agent 4 , representing the chair is kept at $x_{4}=0$ in the left plot as the chair did not participate in the P\&T committee during the decision making process. The contours show how the agents who had made decisions $a$ priori affected the others with pure blue and red colors corresponding to the extreme decisions of +1 and -1 . Within the square region, only $28 \%$ have values $x(\mathbf{z})>0$. In the middle plot, $x_{4}=+1$ to show how the influences change with agent 4's presence and playing an equal role as the other decided agents. The net positive area is now increased to $42 \%$. The plot to the right depicts what would happen if the chair agent assumed a larger role than the other agents, depicting effective leadership. Accordingly, $x_{4}=$ +2 and $\sigma$ is increased to $16 \times 10^{-4}$ for this agent. The area covered by $x(\mathbf{z})>0$ increases significantly to $94 \%$ showing that the presence of an effective chair can play a decisive role in ensuring a fairer outcome.

Although not directly related to this situation, the positive role of effective leadership has been reported els ewhere [17], [18].

\section{Equilibrium}

The decision variable $x_{i}^{0}$ was initialized in accordance with the expression in (7). The value of $\alpha$ was kept low at $\alpha=0.15$, and that of $\varepsilon$ was kept at $\varepsilon=10^{-6}$.

With all other parameters at their earlier values, this study examined the role of inbreeding in influencing the undecided agents. Therefore $\gamma$ was varied between 0 and 1 in steps of 0.2 . The final decision vector was obtained for each value of $\gamma$, as shown in Fig. 2 with $\mu=0.5$ (left), $\mu=1$ (middle), and $\mu=2.0$ (right).

Within each subplot, the decisions of the agents in $\mathcal{V}_{\mathrm{U}}$ appear sorted in order of increasing productivities. Thus, the decision $x_{2}$ of agent $2\left(p_{2}=0.229\right)$ is the leftmost and $x_{5}$, which is that of agent $5\left(p_{5}=3.779\right)$ is the rightmost. Within each histogram, the vertical bars are colored according to the value of $\gamma$. The purple bars correspond to $\gamma=0$, blue bars to $\gamma=0.2$ and so on, until the yellow bars, which are for $\gamma=1$.

The effect of increasing $\gamma$ is clearly seen. As it increases, the decisions $x_{2}$ and $x_{3}$ decrease steadily. This is because agents 2 and 3 , which are in $\mathcal{J}$ are more influenced by malicious agent 11 who is also in $\mathcal{J}$. The decisions of the other agents, agent 5 and agent 9 remain largely unaffected. This decrease is seen for all three values of $\mu$. Moreover, the similarity of the three subplots indicate the robustness of the model to the parameter $\mu$.

\section{CONCLUSION}

In conclusion, this research has been shown to accurately model the outcome seen in a recent $\mathrm{P} \& \mathrm{~T}$ decision process at 
a department. The two-dimensional embedding in Figure 2, which is the outcome of the earlier static study [16], very effectively captured the voting patterns of the undecided agents.

The subsequent equilibrium analysis in this research was able to eliminate the minor discrepancy that persisted between the model's prediction with the observed outcome through the introduction of a small inbreeding coefficient.

Although not shown, preliminary results in applying the model to randomly generated data reveals that the model is able to faithfully reproduce 'common sense' results where more research-oriented faculty vote more consistently and fairly, than less productive ones. It corroborates the observations reported els ewhere [19], [20].

At the present time, the author is investigating if there exists a tipping point in the ratio of unproductive to productive faculty, that when exceeded, leads to anomalous outcomes

\section{REFERENCES}

[1] E. P. Steven, "Using agent-based simulation to examine the robustness of up-or-out promotion systems in universities," Nonlinear Dyn. Psychol. Life Sci, vol. 8, 2004, pp. 177-203.

[2] F. J. Ricardo and G. Monteiro, "The tenure game: Building up academic habits," The Japanese Economic Review, vol. 59, no. 3, 2008, pp. 370-380.

[3] A. Ian, C. Rowat, and N. Zakariya, Optimal Two Stage Committee Voting Rules, Department of Economics, University of Birmingham, 2007.

[4] A. S. Daniel, "Spectral graph theory and its applications," in Proc. $48^{\text {th }}$ Annual IEEE Symposium on Foundations of Computer Science, 2007, 29-38.

[5] G. Olivier and A. Louvet, "Academic inbreeding: An evaluation," Published in Laviedesidees, 2008.

[6] H. Hugo, F. M. Veloso, and R. Grediaga, "Navel gazing: Academic inbreeding and scientific productivity," Management Science, vol. 56 no. 3, 2010, pp. 414-429.

[7] H. Hugo, "Deepening our understanding of academic inbreeding effects on research information exchange and scientific output: new insights for academic based research." Higher Education 65, no. 4 (2013): 487-510.

[8] I. Ozlem and O. Tuncer, "The effect of academic inbreeding on scientific effectiveness," Scientometrics, vol. 88, no. 3, 2011, pp. 885898.

[9] B. Mikhail and P. Niyogi, "Laplacian eigenmaps and spectral techniques for mbedding and clustering," Advances in Neural Information Processing Systems, pp. 585-591, 2002.

[10] B. Yeshua, O. Delalleau, and N. L. Roux, "Label propagation and quadratic criterion," in Book Chapter in Semi-Supenised Learning. Cambridge, MA: MIT Press, 200
[11] F. J. Ricardo, P. A. Loureiro, F. G. Mixon, and A. Sachsida, "Faculty promotion in academe: theory and evidence from US economics departments," Journal of Economics and Econometrics, vol. 56, no. 1, 2013, pp. 1-27.

[12] Y. F. Chen and G. Zoega, Life-Cycle, Effort and Academ ic Deadwood, Economic Studies, University of Dundee, 2010.

[13] N. Nikolaos, A. C. Tsikliras, S. Somarakis, and K. I. Stergiou. "Tenure and academic deadwood," Ethics in Science and Environmental Politics, vol. 15, no. 1, 2015, pp. 87-93.

[14] P. P. Christopher, B. B. Baltes, S. A. Young, J. W. Huff, R. A. Altmann, H. A. Lacost, and J. E. Roberts, "Relationships between psychological climate perceptions and work outcomes: A meta Analytic review," Journal of Organizational Behavior, vol. 24, no. 4, 2003, pp. 389-416.

[15] S. C. Yan, D. Xu, B. Y. Zhang, H. J. Zhang, Q. Yang, and S. Lin, "Graph embedding and extensions: A general framework for dimensionality reduction," IEEE Transactions on Pattem Analysis and Machine Intelligence, vol. 29, no. 1,2007, pp. 40-51.

[16] D. Sanjoy, "Detecting collusive and opportunistic voting strategies in an academic department P\&T decision using low-dimensional spectral embedding," in Proc. International Conference on Big Data Analysis, Shanghai, China, March 2018

[17] American Council on Education, "Good Practice in Tenure Evaluation Advice for Tenured Faculty Department Chairs and Academic Administrators,"2000.

[18] J. B. Carole, B. A. Center, D. A. Finstad, K. R. Risbey, and J. G. Staples, "A theoretical, practical, predictive model of faculty and department research productivity," Academic Medicine, vol. 80, no. 3, 2005 , pp. 225-237.

[19] J. G. G. Connie, J. E. Dutton, and J. M. Bartunek, "Learning from academia: The importance of relationships in professional life," Academy of Management Joumal, vol. 43, no. 6, 2000, pp. 1026-1044.

[20] Z. Natalia and M. Bagues, "The role of connections in academic promotions," American Economic Journal: Applied Economics, vol. 7, no. 2, 2015, pp. 264-292.

Sanjoy Das received his Ph.D. in electrical and computer engineering from Louisiana State University, Bat on Rouge, USA in 1994. Bet ween 1994 and 1997, he received his post-doctoral training at the University of California, Berkeley, USA, where he focused on neural networks and neuro-motor control.

After serving in the industry as a research scientist, he joined the Electrical and Computer Engineering Department at Kansas State University in 2001, where he is currently an Associate Professor. His research interests include machine learning, mathematical aspects of data science, neural net works, multi-agent systems, algorithmic game theory and quant um computing.

Dr. Das has published over 60 journal papers and 100 conference papers. He has served in the technical committees and editorial boards in various international conferences and technical journals. 